\title{
Prediction of Sequential Values for Debt Recovery
}

\author{
Tomasz Kajdanowicz and Przemysław Kazienko \\ Wrocław University of Technology, Wyb. Wyspiańskiego 27, 50-370 Wrocław, Poland \\ \{tomasz.kajdanowicz, kazienko\} @pwr.wroc.pl
}

\begin{abstract}
The concept of new approach for debt portfolio pattern recognition is presented in the paper. Aggregated prediction of sequential repayment values over time for a set of claims is performed by means of hybrid combination of various machine learning techniques, including clustering of references, model selection and enrichment of input variables with prediction outputs from preceding periods. Experimental studies on real data revealed usefulness of the proposed approach for claim appraisals. The average accuracy was over $93 \%$, much higher than for simplifier methods.
\end{abstract}

Keywords: financial pattern recognition, prediction, repayment prediction, claim appraisal, competence regions modeling.

\section{Introduction}

Valuation of the debt portfolio is a prediction task that assesses the possible repayment value from the debt cases. All business models that rely on the cash flow from receivables assume to minimize the aggregated debt value. The fact that possession of creditors for a long time is very ineffective, especially when debtors are eventually not able to repay theirs arrears in short term, implies a need of sophisticated debts valuation. Under some circumstances, it is better for companies to sell the liabilities to a specialized debt collection company in order to obtain at least a part of their nominal value, rather than collect and vindicate debts on their own. In the process of selling a debt portfolio the transaction price is usually estimated based on the possible repayment level to be reached in the long term. In general, it is expected that the method of debt portfolio value appraisal will well match the future.

\section{Related Work}

There exists a wide variety of studies on prediction and classification in the literature e.g. $[2,12,16]$. Overall, the existing machine learning methods usually provide better classification and prediction accuracy than techniques based only on common statistical techniques such as regression $[17,18]$. A better precision of the prediction may be obtained by combination of several existing methods into one hybrid solution $[1,2$, 4]. In general, hybridization could be achieved either by application of additional external mechanisms into existing prediction models (low level), e.g. neuro-fuzzy systems [11] or by combination of different methods on the high level, e.g. multiple 
classifier systems, where separate classifiers are treated more likewise 'black boxes' $[6,7]$. Hybrid prediction methods have been successfully used in a number of domains such as medicine, engineering and industry. Other application areas of these methods are economy and finance, where hybrid systems provide specialized knowledge in order to support business decisions [15].

The paper is focused on the description of a new hybrid method for debt portfolio appraisal. The correct prediction of target repayment value in debt recovery is of great practical importance, because it reveals the level of possible expected benefit and chances to collect receivables. The crucial concept of this method is the combination of clustering of the training set and application of multiple classifiers based on their competence region[12]. Additionally, a sequence of classifiers is built to obtain predictions over consecutive periods. Apart from the general idea, the proposed hybrid prediction method has been examined on real data. According to the findings achieved, the method appears to return more precise results compared to some common approaches.

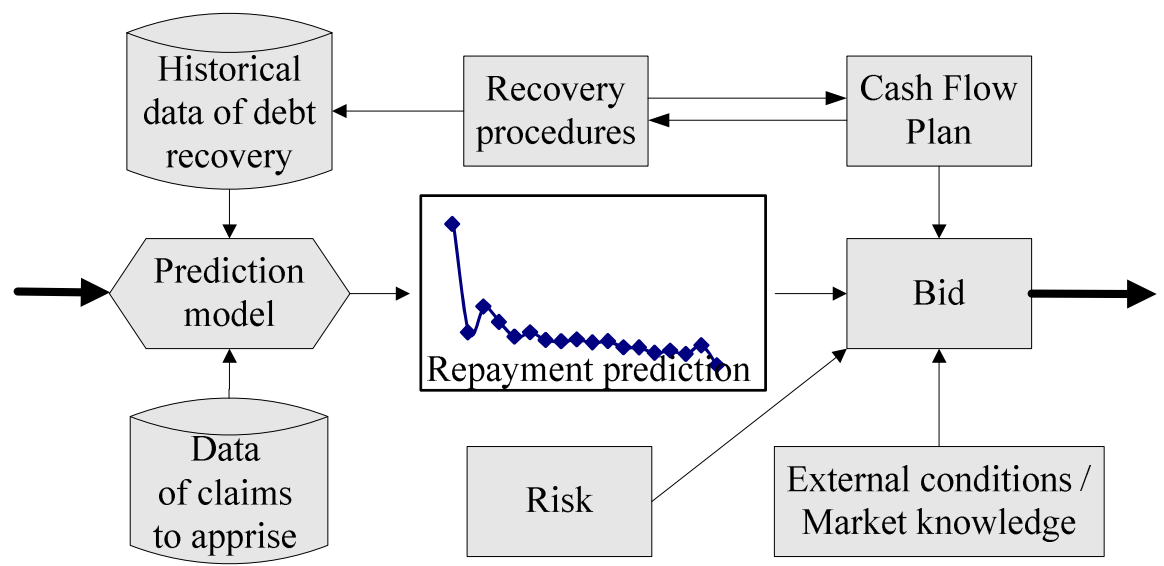

Fig. 1. The business proces of purchasing a debt portfolio based on repayment prediction

\section{Claim Appraisal}

\subsection{Business Process of Debt Portfolio Recovery}

The process of debt portfolio value prediction starts when the first company offers a package of debts and expects a purchase proposal from the second one, see Fig. 1. The second company is usually a specialized debt recovery entity. Based on historical data of debt recovery available for the second company, a prediction model is prepared. The model provides estimation of possible return from the package. The bid is supplemented by additional cost of repayment procedures and cash flow abilities as far as risk and final purchase price are proposed to the first company. The most significant and sensitive part of the process is the repayment value prediction for debt portfolio as there is a strong business need for the method to be designed for efficient and accurate prediction with the time factor. 


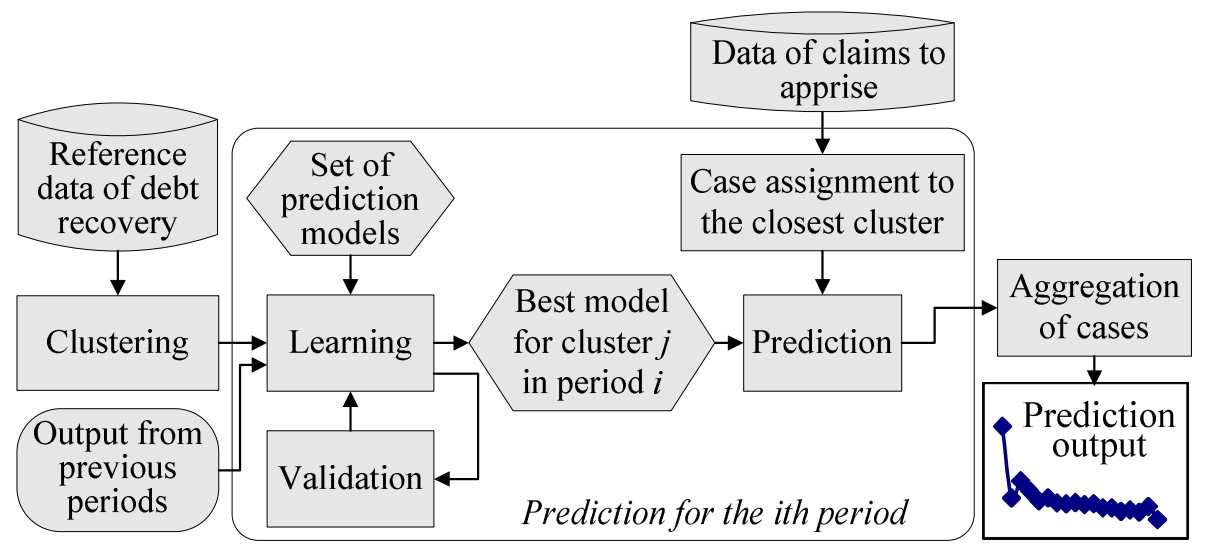

Fig. 2. Concept of sequential prediction of sequential debt profolio valuation

Having the data of historical claim cases together with their repayment profiles over time, a debt collection company can build a model in order to predict receivables for the new claim set invited for bids. However, in order to be able to evaluate cash flows in the following periods (usually months), the company needs to have possibly precise distribution of the receivables collection. It helps to estimate the final upper value for the considered input debt portfolio. Hence, not only the total aggregated value of the receivables is useful for bidding but also their probable timing, period by period.

\subsection{The Concept of the Hybrid Valuation Method}

The idea of the hybrid method for prediction of debt recovery value consists of data flows that are executed separately for each period $i$ ( $M$ times), Fig. 2. First, the prepared historical data is clustered into groups of similar debt cases. Next, a set of models is created (learnt) separately for each cluster $j$ using the fixed set of common, predefined models. The best one is selected for each cluster and becomes the cluster's predictive model $P_{i j}$. This assignment is done based on minimization of the standard deviation error. This is the main learning phase followed by the final prediction for the debt portfolio. For each of debt cases, the closest cluster of historical data is determined and the prediction for this case is performed based on the model assigned and trained on that cluster, separately for each period $i$.

The important characteristic of the method is that the predicted value of return on debt in period $i$ is taken as the input variable for the $i+1$ th period prediction as an additional feature in the model, see Fig. 3.

Historical, reference cases are in general clustered into $N^{G}$ groups using partitioning method and the best prediction model is separately assigned to each group and each period $i$. Features directly available within the input data set or new ones derived from them are the only used in the clustering process. Besides, clustering is performed for the whole reference set, i.e. for cases being after at least one period of the recovery 


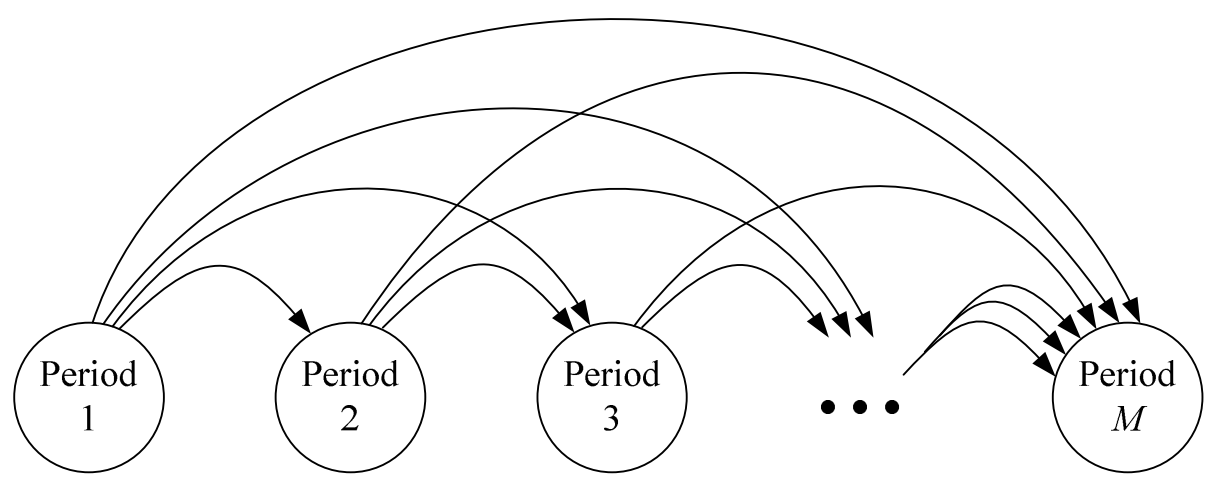

Fig. 3. Input variable dependency in sequential prediction of debt repayment

procedure (period 1). For the following periods, e.g. for period $i$, cases with to short history (being recovered shorter than $i$ periods), are just removed from their clusters without re-clustering. As a result, the quantity of each cluster $G_{i j}$ may vary depending on the period $i$ and it is smaller for greater $i$. For the $j$ th group $G_{i j}$ and the $i$ th period, we have: $\operatorname{card}\left(G_{i j}\right) \geq \operatorname{card}\left(G_{(i+1) j}\right)$. In consequence, there are the same reference groups for all periods but their content decreases for the following periods. This is obvious, because the debt collection company possesses many pending recovery cases, which can be used as references in prediction only for the beginning periods. If the quantity of one cluster for the greater period is too small than this cluster is merged with another, close one for all following periods.

Each group $G_{i j}$ possesses its own representation and the common similarity function is used to evaluate closeness between group $G_{i j}$ and each input case $x$ just being predicted. Next, the single closest group, or more precise the assigned model, is applied to the input case $x$.

\section{Experimental Setup}

For the experimental examination of the proposed method 12 distinct real debt recovery data sets were used. A summary of the data profile is presented in Tab. 1. In total, 20 input features were extracted: 5 continuous, 9 nominal and 6 binary. The experiments were performed using 5 cross-fold validation setup independently applied for each data set [5]. As the proposed prediction process consists of algorithms which efficiency depends on some parameters, some preliminary assessments were applied. The key parameters of the hybrid method are: the number of groups that the clustering process produces, the number and types of predictors used and the method for the selection of the best predictor for each group. The number of groups was adjusted from the range of 5 to 50 by means of X-means algorithm [14]. The average number of groups was 17.6. Three simple predictors were used: M5P tree, logistic regression and regression tree. Decision of taking these relatively simple machine learning approaches was caused by the high computational cost of 
prediction for each group and each period. The total number of predictions for only one data set was: 17.6 groups $* 10$ periods $* 3$ predictors $* 5$ cross-validations $=$ 2640 models to be learnt. For 12 data sets, altogether 31680 predictors were exploited. Obviously, the usage of more complex and sophisticated prediction methods is envisaged in future research. The best predictor assignment to each group is carried out based on the minimization of the prediction standard deviation. The research was implemented and conducted within the $\mathrm{R}$ statistical computing environment with the extended and customized algorithms based on RWeka, rJava and tree plug-ins.

In the experiment, the debt recovery value prediction has been conducted for 10 consecutive periods (months). Three different scenarios were realized and finally compared with each other. In each scenario, the output of period $i$ is used as the input variable for the following periods. The first scenario assumes simple prediction to be carried out on the single model (regression tree), which is learnt and validated on the training data without clustering. The learning is accomplished separately for each period. The first scenario can be treated as the basic approach for value prediction of sequential and continuous variables. In the second scenario, also without clustering, the assessment of three distinct predictors is performed and the best one is chosen for each period. The full hybrid process is performed in the third scenario, including clustering of the reference data set, see Fig. 3 and 4. Clustered data was used to train all models and the best model was determined for each cluster. Next, in the testing phase, the input cases were assigned to the closest cluster and processed by the assigned predictor. In other words, if an appraisal case is close to a certain cluster, the return value would be predicted by the model assigned to that cluster. The second scenario extends the first one, whereas the third expands the second.

Table 1. Summary of debt recovery data sets

\begin{tabular}{|c|c|c|c|c|c|c|c|}
\hline $\begin{array}{c}\text { Data } \\
\text { set }\end{array}$ & $\begin{array}{c}\text { Number } \\
\text { of cases }\end{array}$ & $\begin{array}{c}\text { Data } \\
\text { set }\end{array}$ & $\begin{array}{c}\text { Number } \\
\text { of cases }\end{array}$ & $\begin{array}{c}\text { Data } \\
\text { set }\end{array}$ & $\begin{array}{c}\text { Number } \\
\text { of cases }\end{array}$ & $\begin{array}{c}\text { Data } \\
\text { set }\end{array}$ & $\begin{array}{c}\text { Number } \\
\text { of cases }\end{array}$ \\
\hline A & 4019 & D & 3175 & G & 6818 & J & 6607 \\
\hline B & 3440 & E & 3736 & H & 1703 & K & 2515 \\
\hline C & 2764 & F & 4211 & I & 4584 & L & 1104 \\
\hline
\end{tabular}

\section{Experimental Results}

Having established the methods for debt appraisal, three scenarios were launched and compared with each other in respect of average prediction accuracy. The results of experiments are presented in Tab. 2.

The results of three distinct prediction scenarios revealed that the third scenario (the comprehensive, hybrid approach) performs better by $23 \%$ than the first one (basic prediction for sequential, continuous values with the single predictor) and by 
Table 2. The results of debt recovery value prediction for three different scenarios

\begin{tabular}{|c|c|c|c|}
\hline \multirow[b]{2}{*}{ Data set } & \multicolumn{3}{|c|}{ Prediction accuracy } \\
\hline & $\begin{array}{l}\text { Scenario1: single } \\
\text { predictor }\end{array}$ & $\begin{array}{c}\text { Scenario 2: } \\
\text { predictor selection }\end{array}$ & $\begin{array}{c}\text { Scenario3: predictor } \\
\text { selection with clustering }\end{array}$ \\
\hline A & $64.56 \%$ & $67.03 \%$ & $95.11 \%$ \\
\hline $\mathrm{B}$ & $53.48 \%$ & $60.40 \%$ & $88.26 \%$ \\
\hline $\mathrm{C}$ & $68.98 \%$ & $89.51 \%$ & $93.49 \%$ \\
\hline $\mathrm{D}$ & $70.35 \%$ & $80.52 \%$ & $88.98 \%$ \\
\hline$E$ & $73.50 \%$ & $73.72 \%$ & $92.20 \%$ \\
\hline$F$ & $69.40 \%$ & $95.56 \%$ & $95.99 \%$ \\
\hline G & $95.59 \%$ & $96.24 \%$ & $96.63 \%$ \\
\hline $\mathrm{H}$ & $79.51 \%$ & $92.02 \%$ & $96.11 \%$ \\
\hline I & $45.81 \%$ & $87.97 \%$ & $89.83 \%$ \\
\hline $\mathrm{J}$ & $71.33 \%$ & $86.10 \%$ & $98.89 \%$ \\
\hline $\mathrm{K}$ & $68.07 \%$ & $81.95 \%$ & $92.14 \%$ \\
\hline $\mathrm{L}$ & $84.25 \%$ & $91.22 \%$ & $93.15 \%$ \\
\hline $\begin{array}{l}\text { Average } \\
\text { accuracy }\end{array}$ & $70.40 \%$ & $83.52 \%$ & $93.40 \%$ \\
\hline
\end{tabular}

$10 \%$ better than the second (with the best predictor selection). The third final method for debt portfolio valuation stays in high contrast with other simpler approaches, especially as regards the prediction accuracy as well as prediction error stability, see Tab. 4.

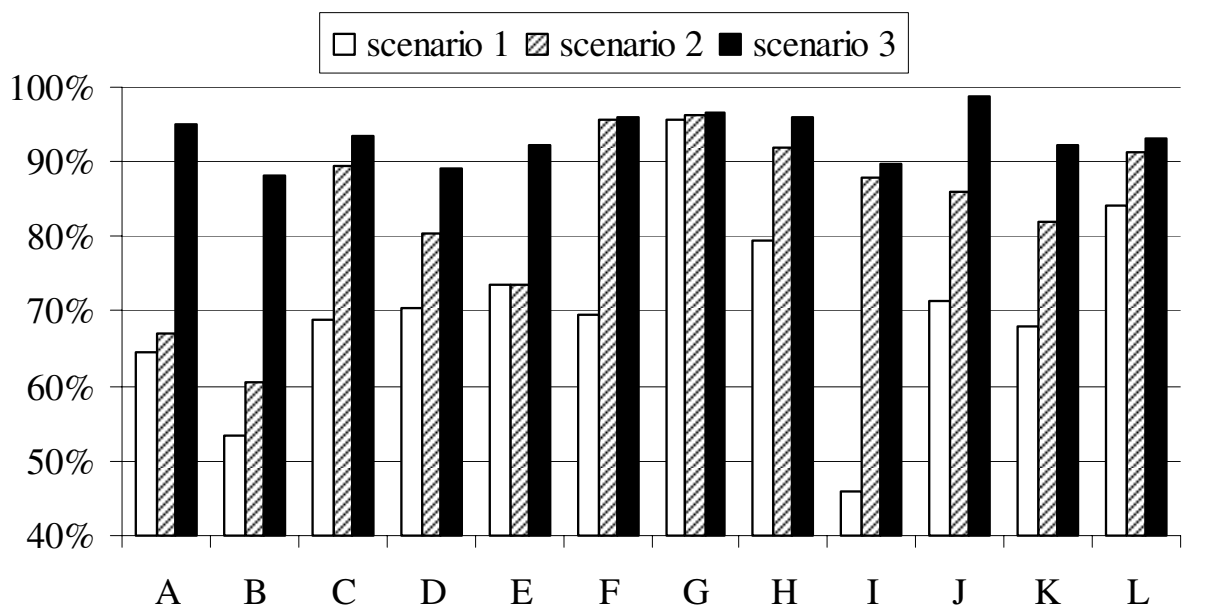

Fig. 4. The accuracy of debt recovery value prediction for three different scenarios for each data set from A to $\mathrm{L}$ 
Studying ensemble like approaches, it is worth analyzing the error performance in terms of bias and variance factors. The bias and variance reflects the contribution of the prediction error for consecutive periods to the general error prediction [8]. Although, it may happen that the aggregated value of prediction for all periods reveals smaller error rate than the sum of errors from all periods, the prediction for the single period may overestimate or underestimate. Bias and variance of the third scenario prediction is presented in the Fig. 5.

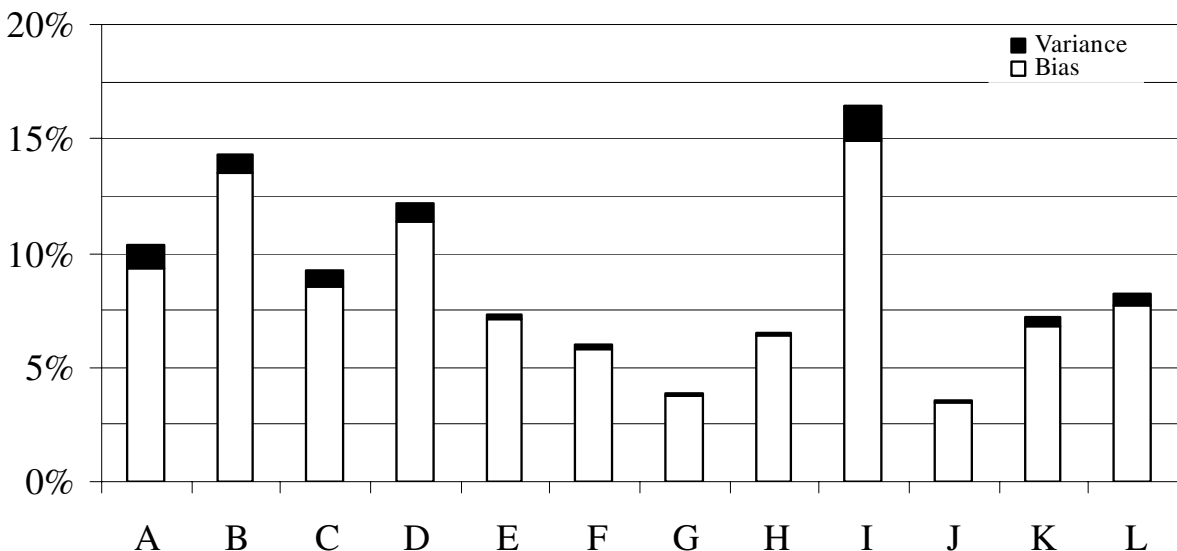

Fig. 5. Bias / variance decomposition for sequential (period) prediction error

As seen in Fig. 5, the stability with respect to prediction error over time is directly reflected in low variance term, concerning most of the error in the bias.

\section{Conclusions and Future Work}

In order to predict debt portfolio value, the proper hybrid method has been suggested and examined on real data. The experimental results support the conclusion that combined prediction solutions are more accurate and may be efficiently applied to debt recovery valuation.

In the future studies, many further aspects improving the method will be considered, in particular: combination of distinct types of classifiers, models' tuning using genetic based optimization [13] and adaptive clustering.

The application of the similar hybrid concept is also considered to be applied to social-based recommender systems [10].

Acknowledgments. The work was supported by The Polish Ministry of Science and Higher Education, the development project, 2009-11. 


\section{References}

1. Aburto, L., Weber, R.: A Sequential Hybrid Forecasting System for Demand Prediction. In: Perner, P. (ed.) MLDM 2007. LNCS (LNAI), vol. 4571, pp. 518-532. Springer, Heidelberg (2007)

2. Ali, S., Smith, K.: On learning algorithm selection for classification. Applied Soft Computing 6(2), 119-138 (2006)

3. Bishop, C.M.: Pattern Recognition and Machine Learning. Springer, Heidelberg (2006)

4. Chou, C.-H., Lin, C.-C., Liu, Y.-H., Chang, F.: A prototype classification method and its use in a hybrid solution for multiclass pattern recognition. Pattern Recognition 39(4), 624 634 (2006)

5. Dietterich, T.G.: Approximate statistical tests for comparing supervised classification learning algorithms. Neural Computation 10(7), 1895-1923 (1998)

6. Eastwood, M., Gabrys, B.: Building Combined Classifiers, A chapter in Knowledge Processing and Reasoning for Information Society. In: Nguyen, N.T., Kolaczek, G., Gabrys, B. (eds.), pp. 139-163. EXIT Publishing House, Warsaw (2008)

7. Gabrys, B., Ruta, D.: Genetic algorithms in classifier fusion. Applied Soft Computing 6(4), 337-347 (2006)

8. Garcia-Pedrajas, N., Ortiz-Boyer, D.: Boosting k-nearest neighbor classifier by means of input space projection. Expert Systems with Applications 36, 10570-10582 (2009)

9. Kajdanowicz, T., Kazienko, P.: Hybrid Repayment Prediction for Debt Portfolio. In: ICCCI 2009. LNCS (LNAI), vol. 5796, pp. 850-857. Springer, Heidelberg (2009)

10. Kazienko, P., Musiał, K., Kajdanowicz, T.: Multidimensional Social Network and Its Application to the Social Recommender System. IEEE Transactions on Systems, Man, and Cybernetics - Part A: Systems and Humans (in press, 2009)

11. Keles, A., Kolcak, M., Keles, A.: The adaptive neuro-fuzzy model for forecasting the domestic debt. Knowledge-Based Systems 21(8), 951-957 (2008)

12. Kuncheva, L.: Combining Pattern Classifiers. Methods and Algorithms. John Wiley \& Sons, Inc., Chichester (2004)

13. Lin, P.-C., Chen, J.-S.: A genetic-based hybrid approach to corporate failure prediction. International Journal of Electronic Finance 2(2), 241-255 (2008)

14. Pelleg, D., Moore, A.W.: X-means: Extending K-means with Efficient Estimation of the Number of Clusters. In: International Conference on Machine Learning, pp. 727-734. Morgan Kaufmann Publishers Inc., San Francisco (2000)

15. Ravi, V., Kurniawan, H., Nwee, P., Kumar, R.: Soft computing system for bank performance prediction. Applied Soft Computing 8(1), 305-315 (2008)

16. Rud, O.: Data Mining Cookbook. Modeling Data for Marketing, Risk, and Customer Relationship Management. John Wiley \& Sons, Inc., Chichester (2001)

17. Swanson, N.R., White, H.: A model selection approach to assessing the information in the term structure using linear model and the artificial neural network. Journal of Business and Economics Statistics 13, 265-275 (1995)

18. Zurada, J., Lonial, S.: Comparison of The Performance of Several Data Mining Methods For Bad Debt Recovery In The Healthcare Industry. The Journal of Applied Business Research 21(2), 37-53 (2005) 